\title{
An Improved Type-2 Possibilistic Fuzzy C-Means Clustering Algorithm with Application for MR Image Segmentation
}

\author{
Xiangjian Chen ${ }^{1}, \mathrm{Di} \mathrm{Li}^{2}$ and Hongmei $\mathrm{Li}^{1 *}$ \\ ${ }^{1}$ Jiangsu university of science and technology, School of computer science and \\ Engineering, China \\ ${ }^{2}$ China Shipbuilding Industry corporation 723, China \\ *cxj831209@163.com
}

\begin{abstract}
This paper presents a new clustering algorithm named improved type-2 possibilistic fuzzy c-means (IT2PFCM) for fuzzy segmentation of magnetic resonance imaging, which combines the advantages of type 2 fuzzy set, the fuzzy c-means (FCM) and Possibilistic fuzzy c-means clustering (PFCM). First of all, the type 2 fuzzy is used to fuse the membership function of the two segmentation algorithms (FCM and PCM), the membership function is an interval distribution, the determined fuzzy values which are the outputs of the FCM and PCM. Secondly, the initialization of cluster center and the process of type-reduction are optimized in this algorithm, which can greatly reduce the calculation of IT2PFCM and accelerate the convergence of the algorithm. Finally, experimental results are given to show the effectives of proposed method in contrast to conventional FCM, PFCM and type 2 fuzzy c-means.
\end{abstract}

Keywords: Fuzzy c-means (FCM), Possibilistic fuzzy c-means clustering (PFCM), improved type-2 possibilistic fuzzy c-means (IT2PFCM), magnetic resonance imaging

\section{Introduction}

Using of digital techniques for processing has become necessary in the applications of medical imaging with the increasing number of medical images. A lot of techniques are used for diagnosing images such as magnetic resonance imaging [1-5] (MRI), computed tomography (CT) and so on. Many researchers and doctors used MR images because MRI has some superiority over other diagnostic imaging methods.

Many algorithms for magnetic resonance imaging segmentations have been presented. In general, the fuzzy C-means algorithm (FCM) [7-12] is more effective.FCM allows pixels to have relation with multiple clusters with varying degrees of membership. The conventional FCM algorithm is quite difficult to deal with patterns with different volumes. And FCM-based algorithms are all based on type-1 fuzzy set, which could not handle image pattern uncertainties well. The crisp membership functions (MF) used in type-l-set-based FCM has limitation on expressing the uncertainties in images and algorithms. The type- 2 fuzzy set has a secondary membership to define the possibilities of uncertainties in primary membership. Thus the performance of handling uncertainties of type- 2 fuzzy set is enhanced [14-16]. Another similar technique, PFCM [13], proposed interpreted clustering as a possibilistic partition. However, it caused clustering being stuck in one or two clusters.

${ }^{*}$ Corresponding Author 
In this paper, we present a modified type-2 possibilistic fuzzy C-means algorithm, which combines the advantages of type 2 fuzzy set, FCM and PCM, at the same time, The initialization of cluster center and the process of type-reduction are optimized in this algorithm, which can greatly reduce the calculation of interval type-2 FCM and accelerate the convergence of the algorithm.

\section{Background Information}

\subsection{Fuzzy C-Means Clustering Algorithm}

FCM is the most popular fuzzy clustering algorithm. It assumes that number of clusters ' $c$ ' is known in priori and minimizes the objective function as:

$O_{F C M}=\sum_{i=1}^{c} \sum_{k=1}^{n} u_{i k}^{m} d_{i k}^{2}$

Where $d_{i k}=\left\|x_{k}-v_{l}\right\|$ and $u_{i k}$ is the membership of pixel ' $x_{k}$ ' in clustering 'i', which satisfies the following relationship:

$$
\sum_{i=1}^{c} u_{i k}=1 ; i=1,2, \cdots, n
$$

Here ' $\mathrm{m}$ ' is a constant, known as the fuzzifier which controls the fuzziness of the resulting partition. Any norm $\|$.$\| can be used for calculation d_{i k}$. Minimization of $O_{F C M}$ is performed by a fixed point iteration scheme known as the alternating optimization technique. The conditions for local extreme for (1) and (2) are derived using lagrangian multipliers:

$$
u_{i k}=\frac{1}{\sum_{j=1}^{c}\left(\frac{d_{i k}}{d_{j k}}\right)^{\frac{2}{m-2}}} \quad \forall k, i
$$

Where $1 \leq i \leq c, 1<k<n$ and

$$
v_{i k}=\frac{\sum_{k=1}^{n}\left(u_{i k}^{m} x_{k}\right)}{\sum_{k=1}^{n}\left(u_{i k}^{m}\right)} \quad \forall i
$$

The FCM algorithm iteratively optimizes $O_{F C M}(U, V)$ with continuous update of $\mathrm{U}$ and V, FCM fine disposes images which are not corrupted with noise but if the image is not or distorted then it wrongly classifies noisy pixels because of its abnormal feature data which is pixel intensity in the case of images and results in an incorrect membership proper segmentation.

\subsection{Possibilistic Fuzzy C-Means Clustering}

Pal et. al., (2005) proposes the fuzzy approach with the possibilities approach, it has two types of memberships, viz. a possibilitic $\left(t_{k i}\right)$ membership that measures the absolute degree of typicality of a point in any particular cluster and a fuzzy membership $\left(u_{k i}\right)$ that measures the relative degree of sharing point along the clusters. PFCM minimizes the objective functions as: 
$O_{F C M}(U, V, T)=\sum_{i=1}^{c} \sum_{k=1}^{n}\left(a u_{i k}^{m}+b t_{k i}^{n}\right) d_{i k}^{2}+\sum_{i=1}^{c} \gamma_{k} \sum_{k=1}^{n}\left(1-t_{k i}\right)^{\eta}$

Subject to the constraint that

$\sum_{i=1}^{c} u_{k i}=1 \quad \forall i$

Here, $a>0, b>0, m>1$, and $\eta>1$. The constants ' $\mathrm{a}$ ' and ' $\mathrm{b}$ ' define the relative importance of fuzzy membership and typicalility values in the objective function. The minimization of objective function gives the following conditions:

$$
u_{i k}=\frac{1}{\sum_{j=1}^{c}\left(\frac{d_{i k}}{d_{j k}}\right)^{\frac{2}{m-1}}} \quad \forall k, i
$$

And

$$
t_{i k}=\frac{1}{1+\left(\frac{b}{\gamma_{k} d_{k i}^{2}}\right)^{\frac{1}{\eta-1}}} \quad \forall k, i
$$

And

$v_{i k}=\frac{\sum_{k=1}^{n}\left(a u_{i k}^{m}+b t_{k i}^{\eta}\right) x_{k}}{\sum_{k=1}^{n}\left(a u_{i k}^{m}+b t_{k i}^{\eta}\right)} \quad \forall k$

Though PFCM is found to perform better than FCM and PCM but when two highly unequal sized clusters with outliers are given, it fails to give desired results.

\subsection{Type-2 Fuzzy C-Means Algorithm}

Both the FCM and IT2FCM algorithms are objective function based clustering methods used to minimize the distance between a pattern and cluster prototype. Similar to FCM, the IT2FCM is an iterative optimization algorithm that minimizes the objective function as:

$O_{T 2 F C M}(U, V)=\sum_{i=1}^{c} \sum_{k=1}^{n} u_{i k}^{m} d_{i k}^{2}$

Where fuzzifier $m$ is replaces by $m_{1}, m_{2}$ which represent different fuzzy degrees and give different objective functions. To minimize the objective function $O_{T 2 F C M}$, we have

$$
\mu_{i k}=\frac{1}{\sum_{j=1}^{c}\left(\frac{d_{i k}}{d_{j k}}\right)^{\frac{2}{m-1}}} \quad \forall k, i
$$

Where $d_{i k}^{2}=\left\|x_{k}-v_{i}\right\|$ is the distance between input patterns $x_{k}$ and cluster centers $v_{i}$ 
The T2FCM, upper and lower memberships are employed to manage uncertainty for fuzzifier $m$, FOU of fuzzifier $m$ is created by two different fuzzifiers $m_{1}, m_{2}$. Which can be derived from above equal as:

$$
\begin{aligned}
& \bar{\mu}_{i k}= \begin{cases}\frac{1}{\sum_{j=1}^{c}\left(\frac{d_{i k}}{d_{j k}}\right) \frac{2}{m_{1-1}},} & \sum_{j=1}^{c}\left(\frac{d_{i k}}{d_{j k}}\right)<c \\
\frac{1}{\sum_{j=1}^{c}\left(\frac{d_{i k}}{d_{j k}}\right) \frac{2}{m_{2-1}}}, & \text { otherwise }\end{cases} \\
& \underline{\mu}_{i k}= \begin{cases}\frac{1}{\sum_{j=1}^{c}\left(\frac{d_{i k}}{d_{j k}}\right) \frac{2}{m_{1-1}}}, & \sum_{j=1}^{c}\left(\frac{d_{i k}}{d_{j k}}\right) \geq c \\
\frac{1}{\sum_{j=1}^{c}\left(\frac{d_{i k}}{d_{j k}}\right) \frac{2}{m_{2-1}}}, & \text { otherwise }\end{cases}
\end{aligned}
$$

Different from FCM, the output of IT2FCM algorithm is an interval type-2 fuzzy set which cannot be transformed to crisp set by defuzzifier directly. Type-reduction is the first and important step of output processing that computes the centroid of a type-2 fuzzy set and reduces type-2 fuzzy set to type-1 fuzzy set. Centroid type-reducer is employed here for getting accurate cluster centers and desirable clustering results. Afterwards a centroid defuzzifier is used to obtain a crisp center from the type-reduced interval type-1 fuzzy set.

\section{Improved Type-2 Possibilistic Fuzzy C-Means Clustering Algorithm}

From the above discussion, we can see the FCM algorithm are greatly influenced by the noise and outliers, and usually singular value of the individual could lead to a cluster center migration, insufficient is improved by the PFCM, but which is easy to lead to the too much infrared image segmentation, so using the algorithm of FCM or PFCM alone does not obtain a good segmentation results. Here we do not use any of these membership functions as a result, but adopting the advantage of type 2 fuzzy fusion of the FCM and the PFCM, trying to get good segmentation effect.

We define the lower membership of type 2 fuzzy sets is $\underline{\mu}_{X}\left(x_{k}\right)=\min \left(\mu_{i k}(F C M), \mu_{i k}(P C M)\right)$, and the upper membership of type 2 fuzzy sets is $\bar{\mu}_{X}\left(x_{k}\right)=\max \left(\mu_{i k}(F C M), \mu_{i k}(P C M)\right)$, then the first membership function is represented by:

$J_{x k}\left[\bar{\mu}_{X}\left(x_{k}\right), \underline{\mu}_{X}\left(x_{k}\right)\right]$

Then the membership value is no longer $\mu_{i k}(F C M)$ or $\mu_{i k}(P C M)$, between there is the rationality of the existence while the values between interval, such an approach is not simply abandon those who failed to reflect the reasonable value, but from the perspective of a more generalized the accuracy of the clustering algorithm is studied. 
Considering from the complexity of the calculation, we choose type 2 fuzzy, based on the definition of type 2 fuzzy, the second membership functions is 1, clustering center $v_{i}$ has the unity formulation, then the clustering center of the proposed method is :

$v_{X}=\left[v_{l}, v_{R}\right]=\sum_{\mu\left(x_{1}\right) \in J_{x_{1}}} \cdots \sum_{\mu\left(x_{N}\right) \in J_{x_{N}}} \frac{\sum_{k=1}^{N} \mu_{i k}^{m} x_{k}}{\sum_{k=1}^{N} \mu_{i k}^{m}}$

Type-reduction is the first and important step of output processing that computes the centroid of a type-2 fuzzy set and reduces type-2 fuzzy set to type-1 fuzzy set.

\subsection{The Determination of the Initial Clustering Center}

The initial clustering center is selected random in traditional clustering algorithms, which easily lead to converge to local extremum points in clustering process, at the same time increase the number of iterations of the algorithm. References puts forward a method to calculate the initial clustering center, but these methods are for the type 1 fuzzy clustering, which is unable to improve the efficiency of the algorithm when used in T2FCM, because the initial clustering center algorithm is isolated from reduction process in T2FCM. Here is the simple method introduced to calculate the initial clustering center combined with type reduction algorithm:

Step1: Ordering the sample data of the first $\mathrm{j}$ characteristic value in ascending, coordinating the position of the rest characteristic value, then the sample is labeled as $X_{j}$ after coordination ;

Step2 : Calculating the clustering center of sample $X_{j}$ :

$$
V_{j}=\left[v_{1}, \cdots v_{i}\right]=\left[\begin{array}{cc}
\frac{1}{[N / c]} \sum_{k=1}^{[N / c]} x_{k j} \\
\vdots \\
\frac{1}{N-(c-1)[N / c]} \sum_{k=(c-1)[N / c]+1}^{[N / c]} x_{k j}
\end{array}\right]
$$

Step 3: Coordinating the clustering center conclude in Step 2 according to characteristic value of one, calculating the reference clustering center, and clustering center vector of the corresponding feature be arranged according to reference clustering center in the collation, then the initial clustering center is concluded.

Type reduction algorithm is embedded on the initialization process of the operation, after the initialization, the process can skip Step 1 and Step 2 of KM algorithm, which can significantly improve computing speed of the algorithm, so this method is more suitable for the interval type 2 fuzzy clustering algorithm compared with other initialization method

\subsection{Type Reduction Algorithm}

$\mathrm{KM}$ algorithm starts the iterative process using the random initial center, the number of iterations is affected by random center selection and sample size. Reference[] analized the literature of KM algorithm and then EKM algorithm based on empirical data initialization method is proposed. Although EKM can effectively improves the reduction speed, but which did not consider the samples has its own features, so EKM is difficult to deal with cluster analysis in a wide variety samples. This paper initialize the clustering center based 
on the weighted average of sample characteristics, which has good adaption for different types of samples. Because the sorting process of KM algorithm is introduced in the initialization process which can effectively reduce the computational complexity of the algorithm.

\section{Simulation and Results}

Since the ground truth of segmentation for real MR images is not usually available, hence segmentation performance cannot be visually compared and it is impossible to evaluate the segmentation performance qualitatively. However, Brainweb provides a simulated brain database (SBD) including a set of realistic MRI data volumes produced by an MRI simulator. These data enable us to evaluate the performance of various image analysis methods in a setting. A simulated MR image including noise, and no intensity of the image is downloaded from brainweb.

In this section, we describe some experimental results to compare the segmentation performance of FCM, PFCM, T2FCM and proposed IT2PFCM algorithms. We test the algorithms on synthetic image with different noises and different MR slices. The segmented images andvalidity functions are evaluated to compare the degree of effectiveness of four methods. In order to further examine the algorithms' effectiveness, we are considering the real brain MR slice corrupted simultaneously by Gaussian white noise $\mathrm{N}(0,180)$.

The class number of the image is assumed as three, corresponding to grey matter, white matter), and background. Figure 1(a), shows an original slice and (b) shows corrupted slice with noise. Figure 1(c-f), shows the segmentation results obtained by applying FCM, T2FCM, PFCM, IT2PFCM, respectively.

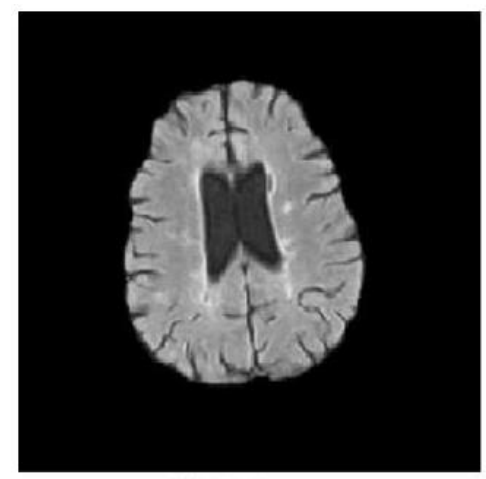

(a) Original Image

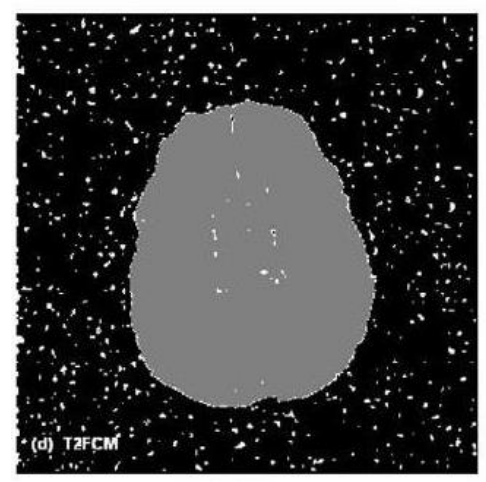

(d) $\mathrm{T} 2 \mathrm{FCM}$

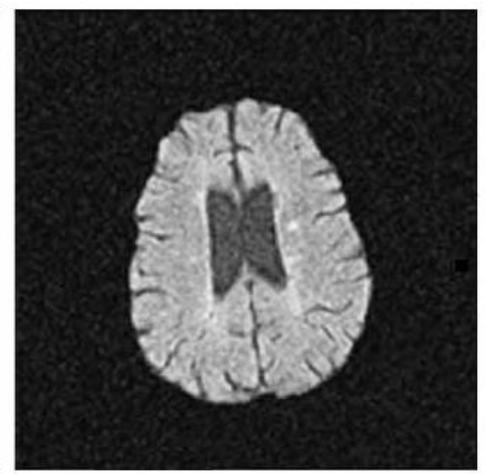

(b) Image with Hoise

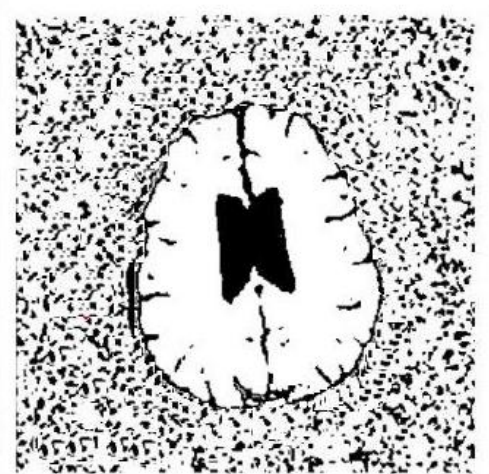

(e) PFCM

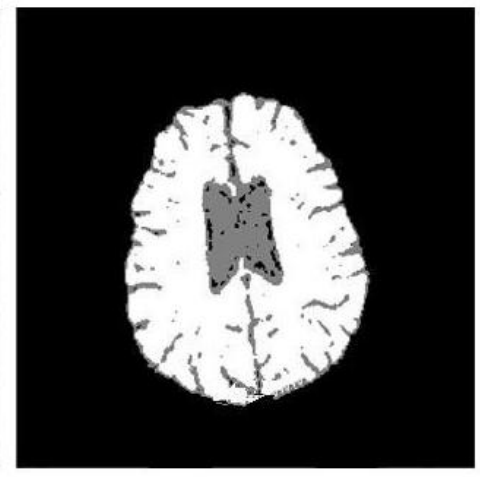

(C) FCM

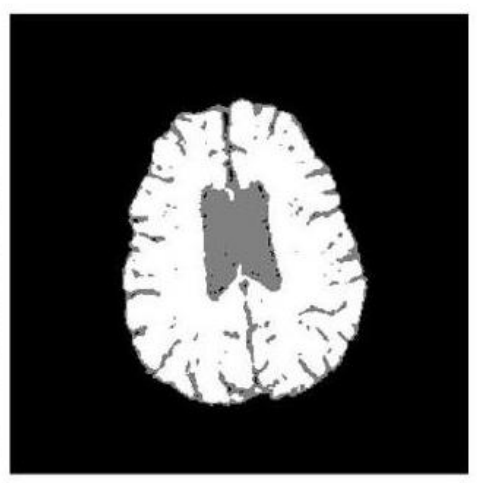

(f) IT2PFCM

Figure 1. Comparison of Segmentation Results on Different MR Images with Gaussian Noise 
As observed from figures, the performance of T2FCM is very bad whereas the performances of all other algorithms are almost similar. It is clearly seen that in the segmentation result of IT2PFCM the noise is suppressed as compared to FCM and PFCM. The result of IT2PFCM is more homogeneous and smoother than other methods.

\section{Acknowledgments}

This paper is supported by National Natural Science Foundation,NO.61502211

\section{References}

[1] M. N. Ahmed, S. M. Yamany, N. Mohamed, A. A. Farag and T. Moriarty, "A modified fuzzy C-means algorithm for bias field estimation and segmentation of MRI data", IEEE Transactions on Medical Imaging, vol. 21, (2002), pp. 193-199.

[2] M. A. Balafar, A. R. Ramli, M. L. Saripan and S. Mashohor, "Review of brain MRI image segmentation methods", Artificial Intelligence Review, vol. 33, (2010), pp. 261-274.

[3] J. C. Bezdek, L. O. Hall and L. P. Clarke, "Review of MR image segmentation techniques using pattern recognition", Medical Physics, vol. 20, (1993), pp. 10-33.

[4] P. M. Birgani, M. Ashtiyani and S. Asadi, "MRI segmentation using fuzzy C-means clustering algorithm basis neural network", In: Third International Conference on ICTTA, Damascus, (2008), pp. 1-5.

[5] M. C. Clark, L. O. Hall, D. B. Goldgof, L. P. Clarke, R. P. Velthuizen and M. S. Silbiger, "MRI segmentation using fuzzy clustering techniques", IEEE Engineering in Medicine and Biology Magazine, vol. 13, (1994), pp. 730-742.

[6] L. P. Clarke, R. P. Velthuizen, M. A. Camacho, J. J. Heine, M. Vaidyanathan, L. O. Hall, R. W. Thatcher and M. L. Silbiger, "MRI segmentation: methods and applications", Magnetic Resonance Imaging, vol. 13, (1995), pp. 343-368.

[7] Y. Fukuyama and M. Sugeno, "A new method of choosing the number of clusters for the fuzzy C-means method", In: Proceedings of Fifth Fuzzy Systems symposium, (1989), pp. 247-250.

[8] J. J. Tang, G. H. Zhang, Y. H. Wang, H. Wang and F. Liu, "A hybrid approach to integrate fuzzy Cmeans based imputation method with genetic algorithm for missing traffic volume data estimation", Transportation Research Part C: Emerging Technologies, vol. 51, (2015), pp. 29-40.

[9] Z. X. Ji, Y. Xia, Q. S. Sun and G. Cao, "Interval-valued possibilistic fuzzy C-means clustering algorithm", Fuzzy Sets and Systems, vol. 253, (2014), pp. 138-156.

[10] M. R. P. Ferreira, "Kernel fuzzy c-means with automatic variable weighting", Fuzzy Sets and Systems, vol. 237, (2014), pp. 1-46.

[11] Z. X. Ji, Y. Xia, Q. S. Sun and G. Cao, "Robust spatially constrained fuzzy c-means algorithm for brain MR image segmentation”, Pattern Recognition, vol. 47, (2014), pp. 2454-2466.

[12] A. M. Abdulshahed, A. P. Longstaff, S. Fletcher and A. Myers, "Thermal error modelling of machine tools based on ANFIS with fuzzy c-means clustering using a thermal imaging camera", Applied Mathematical Modelling, (2014).

[13] Z. X. Ji, Q. S. Sun and D. S. Xia, “A modified possibilistic fuzzy c-means clustering algorithm for bias field estimation and segmentation of brain MR image", Computerized Medical Imaging and Graphics, vol. 35, (2011), pp. 383-397.

[14] C. Y. Qiu, J. Xiao, L. Yu, L. Han and M. N. Iqbal, “A modified interval type-2 fuzzy C-means algorithm with application in MR image segmentation", Pattern Recognition Letters, vol. 34, (2013), pp. 13291338

[15] P. Melin and O. Castillo, "A review on type-2 fuzzy logic applications in clustering, classification and pattern recognition", Applied Soft Computing, vol. 21, (2014), pp. 568-577.

[16] D. Enke and N. Mehdiyev, "Type-2 Fuzzy Clustering and a Type-2 Fuzzy Inference Neural Network for the Prediction of Short-term Interest Rates", Procedia Computer Science, vol. 20, (2013), pp. 115-120. 
International Journal of Signal Processing, Image Processing and Pattern Recognition Vol. 9, No. 11, (2016) 